\title{
Simulations of closed-loop wavefront reconstruction for multiconjugate adaptive optics on giant telescopes
}

\section{Brent L. Ellerbroek, Curtis R. Vogel}

Brent L. Ellerbroek, Curtis R. Vogel, "Simulations of closed-loop wavefront reconstruction for multiconjugate adaptive optics on giant telescopes," Proc. SPIE 5169, Astronomical Adaptive Optics Systems and Applications, (24 December 2003); doi: 10.1117/12.506580

SPIE Event: Optical Science and Technology, SPIE's 48th Annual Meeting, 2003, San Diego, California, United States 


\title{
Simulations of closed-loop wavefront reconstruction for multi-conjugate adaptive optics on giant telescopes
}

\author{
Brent L. Ellerbroek ${ }^{a}$ and C. R. Vogel ${ }^{b}$ \\ ${ }^{a}$ AURA New Initiatives Office, 950 N. Cherry Ave., Tucson AZ, 85719 \\ ${ }^{b}$ Montana State University, Dept. of Mathematical Sciences, Bozeman, MT 59717-2400
}

\begin{abstract}
The multi-conjugate adaptive optics (MCAO) systems proposed for future giant telescopes will require new, computationally efficient, concepts for wavefront reconstruction due to their very large number of deformable mirror (DM) actuators and wavefront sensor (WFS) measurements. Preliminary versions of such reconstruction algorithms have recently been developed, and simulations of MCAO systems with 9000 or more DM actuators and 33000 or more WFS measurements are now possible using a single desktop computer. However, the results obtained to date are limited to the case of open-loop wavefront reconstruction, and more work is needed to develop computationally efficient reconstructors for the more realistic case of a closed-loop MCAO system that iteratively measures and corrects time-varying wavefront distortions. In this paper, we describe and investigate two reconstruction concepts for this application. The first approach assumes that knowledge of the DM actuator command vector and the DM-to-WFS influence matrix may be used to convert a closed-loop WFS measurement into an accurate estimate of the corresponding open-loop measurement, so that a standard open-loop wavefront reconstructor may be applied. The second approach is a very coarse (but computationally efficient) approximation to computing the minimum variance wavefront reconstructor for the residual wavefront errors in a closed-loop AO system. Sample simulation results are presented for both concepts with natural guide star (NGS) AO and laser guide star (LGS) MCAO systems on 8- and 32-meter class telescopes. The first approach yields a stable control loop with closed-loop performance comparable to the open-loop estimation accuracy of the classical minimum variance reconstructor. The second approach is unstable when implemented in a type I servo system.
\end{abstract}

Keywords: Multi-conjugate adaptive optics, wavefront reconstruction, extremely large telescopes

\section{INTRODUCTION}

Astronomical multi-conjugate adaptive optics (MCAO) systems on future giant telescopes will require new approaches for wavefront reconstruction on account of their very high number of wavefront sensor (WFS) subapertures and deformable mirror (DM) actuators, not to mention the wide range of natural- and laser guide star constellations currently under discussion. ${ }^{1-4}$ Conventional techniques for computing (applying) wavefront reconstructors in terms of explicit matrix inversions and multiplications will not be applicable, since their calculation requirements scale as approximately the sixth (fourth) power of the telescope aperture diameter. Fortunately, good progress has recently been made in developing more efficient reconstruction algorithms using advanced techniques from computational linear algebra. ${ }^{5-7}$ Wavefront reconstruction simulations of MCAO systems with 8000 or more DM actuators and 33000 or more WFS measurements are now possible using currently available personal computers, and the results obtained are comparable to what would be achieved with the ideal minimum variance, or "optimal," reconstructor. ${ }^{8}$

However, the results reported to date have been limited to the open loop case, where an idealized MCAO system measures, estimates, and corrects the turbulence-induced phase distortion in an instantaneous snapshot. Futher advances will be needed to model and implement MCAO wavefront reconstruction for the more realistic

Further author information: (Send correspondence to B.L.E)

B.L.E.: E-mail: brent@noao.edu, Telephone: 1 (520) 318-8589

C.R.V: E-mail: vogel@math.montana.edu, Telephone: 1 (406) 994-5332 
closed loop case, where the AO system iteratively measures and corrects time-varying turbulence distortions. The standard minimum variance reconstructor optimized for the open-loop case may perform poorly, or even diverge, when naively applied in a closed-loop AO system.

A variety of existing methods have already been developed for closed loop wavefront reconstruction, but none of these appear to be applicable to the case of a very high-order MCAO system..$^{9-11}$ The classical least-squares wavefront reconstructor (defined as the pseudo-inverse of the DM-to-WFS influence matrix) provides acceptable closed-loop performance for conventional AO systems, but yields disappointing results for MCAO without some type of regularization on account of the large number of very poorly observed wavefront modes. Two possible approaches to regularizing the reconstructor include (i) singular value decomposition ${ }^{12}$ and (ii) using a constrained minimum variance reconstructor to filter the reconstructed phase estimate based upon atmospheric turbulence statistics. ${ }^{9}$ Both of these approaches have achieved good results in closed-loop simulations of MCAO systems with up to approximately $1000 \mathrm{DM}$ actuators, but they scale poorly to larger problems because of the explicit matrix multiplies and inversions that are required to compute the reconstructor. The last observation also applies to Kalman filtering and other predictive filtering techniques. ${ }^{10,11}$

We seek to develop new wavefront reconstruction and control algorithms that are (i) applicable to general MCAO guide star and DM configurations (ii) stable in closed loop, (iii) provide near "optimum" performance, (iv) sufficiently efficient to enable simulations of MCAO on future giant telescopes, and (v) practical in terms of their requirements for calibration measurements, atmospheric measurements, and other a priori data.

This paper summarizes our first progress towards these objectives. We have developed and tested two closed-loop wavefront reconstruction concepts for high-order MCAO systems on future giant telescopes. Both of these methods are modifications to the computationally efficient implementation of the classical minimum variance reconstructor previously developed for the open loop case. The first concept assumes that accurate knowledge is available for the DM-to-WFS influence matrix and the current figure of the DM's, and (in effect) uses this calibration data to convert closed-loop WFS measurements into the corresponding open-loop values. The classical open-loop minimum variance reconstructor is then applied to this data, and the reconstructed wavefront aberrations are temporally filtered before they are fit to DM actuator commands.

The second approach is a coarse (but computationally efficient) approximation to the minimum variance wavefront reconstructor for the residual wavefront errors in a closed-loop AO system. The second-order statistics of these residual wavefront errors, which must be known to compute the regularization term for the minimum variance reconstructor, are approximated by assuming that the only source of error is the temporal latency in the AO control loop.

These two wavefront reconstruction concepts will be referred to as Pseudo-Open Loop Control (POLC) and Approximate Closed-Loop Regularization (ACLR) for the remainder of these paper. The two methods may in fact be "blended" by forming a weighted linear combination of the pair of quadratic cost functions defining the two approaches.

We have implemented and tested these wavefront reconstructors in first-order (linear) simulations of closedloop natural guide star (NGS) AO and laser guide star (LGS) MCAO systems on 8- and 32-meter class telescopes. Each MCAO wavefront reconstruction requires approximately 10 (330) seconds for simulations with $D=8 \mathrm{~m}$ $(D=32 \mathrm{~m})$ on a dual-processor desktop computer, and simulations lasting a few hundred to a few thousand closed loop iterations are feasible with some patience. POLC is stable in closed loop, at least for the case where the calibration data needed to convert closed-loop WFS measurements into the corresponding openloop values are known exactly. Performance is very similar to open-loop simulation results achieved with the classical minimum variance reconstructor, since the wavefront control error due to the latency in the AO control loop is relatively small for the sample astronomical MCAO scenario we have considered. By comparison, the ACLR algorithm diverges in closed loop for both conventional AO and MCAO simulations, although the rate of divergence is slower than for the standard minimum variance reconstructor optimized for the open loop case.

All of the results and observations presented here are based on preliminary simulations that should be considered numerical experiments. More work is needed to formally evaluate the numerical stability of these algorithms and their sensitivity to parameter variations. This can be accomplished by explicity computing the 
transfer- and impulse response functions for each control law, which should be feasible for problems with up to about $1000 \mathrm{DM}$ actuators.

The remainder of the paper is organized as follows. Section 2 reviews the classical open-loop minimum variance estimator and very briefly describes prior work to develop computationally efficient forms of this

algorithm. Section 3 presents our basic Z-transform model for the dynamics of a closed loop AO system and summarizes why the minimum variance estimator derived for open-loop wavefront reconstruction is not necessarily satisfatory, or even stable, for this case. Section 4 describes the two wavefront reconstruction and control approaches we have investigated. Section 5 outlines the sample simulation problems we have considered, and section 6 presents the numerical results obtained. Section 7 is a brief summary.

\section{MINIMUM VARIANCE WAVEFRONT RECONSTRUCTION}

The objective of the minimum variance wavefront reconstruction algorithm is to determine a DM actuator command vector, $a$, that best compensates for an atmospheric turbulence profile, $x$, in the sense of minimizing the mean-square phase variance, $\sigma^{2}$, associated with the residual wavefront error, $\phi$. These quantities are related by the formulas

$$
\begin{aligned}
\phi & =H_{x} x-H_{a} a, \\
\sigma^{2} & =\phi^{T} W \phi .
\end{aligned}
$$

Here $H_{x}$ and $H_{a}$ are the influence matrices that relate the turbulence profile and DM actuator command vector to the resulting wavefront in the aperture plane, and $W$ is a positive semidefinite matrix determined by the shape of the aperture. The reconstruction algorithm is a linear operator $E$ applied to the WFS measurement vector $s$, i.e.

$$
a=E s .
$$

In the open-loop case, the WFS measurement vector $s$ is related to the turbulence profile $x$ by the equation

$$
s=G x+n,
$$

where the matrix $G$ is the turbulence-to-WFS influence matrix, and the vector $n$ is the WFS measurement noise. The above model may be formulated to apply to both conventional AO and MCAO systems. In the latter case, the vectors $a, s$, and $\phi$ are composed of several sub-vectors corresponding to distinct DM's, WFS's, and points in the field-of-view, respectively.

In this notation, the minimum variance reconstructor $E_{*}$ that minimizes the expected value of $\sigma^{2}$ is defined by the condition

$$
E_{*}=\arg \min _{E} J_{m v}(E) \equiv \arg \min _{E}\left\langle\sigma^{2}+k|a|^{2}\right\rangle .
$$

The angle brackets, $\langle\cdots\rangle$, denote an ensemble average over the statistics of the turbulence profile $x$ and the WFS noise vector $n$, and $k$ is a very small regularizing term included to yield a unique value for $E_{*}$.

When Eq.'s (1) through (4) are substituted into the formula for $J_{m v}(E)$, it follows that this merit function is quadradic in the coefficients of the reconstruction matrix. Using standard least squares techniques, the value of the minimum variance reconstructor $E_{*}$ may be evaluated as ${ }^{13}$

$$
\begin{aligned}
& E_{*}=F_{x} E_{x}, \\
& F_{x}=\left(H_{a}^{T} W H_{a}+k I\right)^{-1} H_{a}^{T} W H_{x}, \\
& E_{x}=\left[G^{T}\left\langle n n^{T}\right\rangle^{-1} G+\left\langle x x^{T}\right\rangle^{-1}\right]^{-1} G^{T}\left\langle n n^{T}\right\rangle^{-1} .
\end{aligned}
$$

(We have assumed that the random variables $x$ and $n$ are zero-mean). The matrix $E_{x}$ first obtains an Estimate $\widehat{x}$ of the atmospheric turbulence profile $x$, and the matrix $F_{x}$ then determines the best-Fit actuator commands for this estimate. Other representations of the minimum variance reconstructor are also available, and this 
algorithm has long been a standard technique for evaluating and optimizing the performance of AO and MCAO systems via analysis and simulation. ${ }^{9,14,15}$

For later purposes, we note that an identical wavefront reconstruction is obtained by using the maximum likelyhood (ML) estimate $x_{*}$ of the turbulence profile $x$ if the random variables $x$ and $n$ are assumed to be normally distributed. The probability density functions of these quantities are then defined by

$$
\begin{aligned}
& P_{x}(u)=\frac{1}{\sqrt{(2 \pi)^{N_{x}} \operatorname{det}\left(\left\langle x x^{T}\right\rangle\right)}} \exp \left[-u^{T}\left\langle x x^{T}\right\rangle^{-1} u / 2\right], \\
& P_{n}(v)=\frac{1}{\sqrt{(2 \pi)^{N_{n}} \operatorname{det}\left(\left\langle n n^{T}\right\rangle\right)}} \exp \left[-v^{T}\left\langle n n^{T}\right\rangle^{-1} v / 2\right],
\end{aligned}
$$

where the dimensions of $x$ and $n$ are equal to $N_{x}$ and $N_{n}$, respectively. The ML estimate $x_{*}$ is the value of $u$ that maximizes the joint probability of the turbulence profile and the implied estimate $s-G u$ of the WFS measurement noise $n$. More formally,

$$
\begin{aligned}
x_{*} & =\arg \max _{u} P_{x}(u) P_{n}(s-G u) \\
& =\arg \min _{u} J_{m l}(u) \\
& \equiv \arg \min _{u}\left[u^{T}\left\langle x x^{T}\right\rangle^{-1} u+(s-G u)^{T}\left\langle n n^{T}\right\rangle^{-1}(s-G u)\right],
\end{aligned}
$$

which can be solved using the usual quadratic minimization to obtain

$$
x_{*}=E_{x} s .
$$

Next, the actuator command vector $a$ yielding the best fit to the ML turbulence estimate $x_{*}$ is given by

$$
\begin{aligned}
a & =\arg \min _{u}\left[\left(H_{x} x_{*}-H_{a} u\right)^{T} W\left(H_{x} x_{*}-H_{a} u\right)+k|a|^{2}\right] \\
& =F_{x} x_{*} .
\end{aligned}
$$

These representations for $E_{x}$ and $F_{x}$ will be used in section 4 below as the starting point to develop reconstructors for the closed-loop case.

Severe computational difficulties arise in explicitly evaluating the matrix multiplies and inversions appearing in Eq.'s (6) through (8) for simulations involving future giant telescopes, since the number of adds and multiples required scales as the third power of the number of DM actuators and WFS measurements. This is proportional to the sixth power of the telescope aperture diameter if the density of DM actuators and WFS subapertures is held fixed. Fortunately, more sophisticated methods borrowed from computational linear algebra may be used to implement the minimum variance reconstructor without explicitly evaluating it as a full matrix. Briefly, these algorithms provide efficient solutions to linear systems of the form

$$
A u=B v
$$

without actually inverting the matrix $A$, provided that the matrices $A$ and $B$ possess an appropriate structure (sparse, circulant, or Toeplitz; block matrices with blocks possessing one of these properties; or low-rank perturbations to such matrices). Previous papers have described how the minimum variance reconstructor may be efficiently implemented by successively solving the systems $\widehat{x}=E_{x} s, a=F_{x} \widehat{x}$ using these techniques. ${ }^{5-7,16,17}$ For the purposes of this paper, it is enough to note that (i) such methods have been developed for both natural guide star (NGS) and laser guide star (LGS) MCAO systems, and that (ii) the applicability of sparse matrix methods depends upon finding a good sparse approximation to the term $\left\langle x x^{T}\right\rangle^{-1}$ appearing in Eq. (8). We approximate this matrix as $\alpha C^{T} C$, where $\alpha$ is a scale factor and $C$ is a discrete Laplacian operator that computes the Curvature of the turbulence profile at each point on the propagation grid. 


\section{MODELING THE CLOSED-LOOP CASE}

Although the open-loop minimum variance wavefront reconstructor is frequently used to characterize the ideal performance of an AO system configuration, it is has generally not been used for real-time control in actual AO control loops. In Section 2, the minimum variance reconstructor was derived from the assumptions that (i) the WFS measurement vector $s$ was obtained in open loop, without benefiting from any correction due to the prior command to the DM, and (ii) the DM command vector $a$ is computed from scratch each cycle from a new WFS measurement. Neither of these conditions applies in a closed loop AO system, where a classical model incorporating the impact of the temporal dynamics takes the form

$$
\begin{aligned}
s(z) & =G_{x} x(z)-G_{a} a(z)+n(z), \\
r(z) & =E_{x} s(z), \\
\widehat{x}(z) & =g(z) r(z), \\
a(z) & =F_{x} \widehat{x}(z) .
\end{aligned}
$$

The variable $z$ indicates the application of the z-transform to the time histories of the quantities $s, x, a, n$, and $\widehat{x}$. The term $G_{a} a(z)$ in the first equation represents the partial correction applied to the WFS measurement by the DM command. The new variable $r$ denotes an estimate of the Residual phase errors computed at each cycle by applying the reconstruction matrix $E_{x}$ to the closed-loop WFS measurement. Finally, $g(z)$ is a scalar-valued temporal filter applied to the output of the wavefront reconstructor before the phase estimate $\widehat{x}$ is fit to DM actuator commands and applied to the deformable mirror. Because $g(z)$ is scalar valued the filter could just as well have been applied to the DM actuator commands, but we prefer to filter the phase estimate to maintain consistency with the standard implementation of existing modal AO systems.

The classical minimum variance reconstructor will not necessary perform as expected in this dynamical system. As an elementary example, consider the case where the turbulence profile $x$ and the WFS measurement noise $n$ are fixed, and the temporal filter $g(z)$ is a type I controller that corrects constant errors. In this case, the AO control system will converge to steady state when the condition

$$
E_{x} s(t)=0
$$

is satisfied. For the minimum variance reconstructor as defined in Section 2, this is equivalent

$$
G_{x}^{T}\left\langle n n^{T}\right\rangle^{-1} G_{a} a=G_{x}^{T}\left\langle n n^{T}\right\rangle^{-1}\left(G_{x} x+n\right) .
$$

This is not consistent with the open-loop minimum variance solution, and is in fact completely independent of atmospheric turbulence statistics. A new approach is needed to regularize the wavefront reconstruction algorithm in closed loop.

\section{EFFICIENT APPROACHES TO CLOSED-LOOP WAVEFRONT RECONSTRUCTION}

We have now developed and tested several variants of the classical minimum variance reconstructor with a goal of obtaining improved performance in a closed loop AO system. The emphasis has been to find a computationally efficient algorithm with acceptable performance, as opposed to an optimal solution (e.g., a Kalman filter) that would be impractical to implement for a very high order MCAO system. The following subsections describe the concepts we have considered.

\subsection{Pseudo-open loop control (POLC)}

This approach is based on the assumption that perfect a priori knowledge is available for the DM actuator command vector $a$ and influence matrices $G_{x}$ and $G_{a}$, and that the closed loop control problem can be reduced to the open loop case by appropriately combining the current DM command, the most recent turbulence 
estimate, and current WFS measurement of the residual errors. In analogy with Eq. (11) for the classical minimum variance reconstructor, the cost function $J_{\text {polc }}$ for this concept is defined by the formula

$$
\begin{aligned}
r & =\arg \min _{u} J_{\text {polc }}(u) \\
& \equiv \arg \min _{u}\left\{\left[s+G_{a} a-G_{x}(\widehat{x}+u)\right]^{T}\left\langle n n^{T}\right\rangle^{-1}\left[s+G_{a} a-G_{x}(\widehat{x}+u)\right]+(\widehat{x}+u)^{T}\left\langle x x^{T}\right\rangle^{-1}(\widehat{x}+u)\right\}
\end{aligned}
$$

Heuristically, the term $s+G_{a} a$ appearing above is the estimate of the open-loop WFS measurement corresponding to the closed-loop measurement $s$. The term $\widehat{x}+u$ is the estimate for the current turbulence profile after the previous estimate $\widehat{x}$ is updated by the estimate $u$ of the current residual error. Eq. (21) is consequently the direct implemention of the classical minimum variance (or ML) reconstructor in a closed-loop AO system.

To solve Eq. (21) for the residual error $r$, it is convenient to express the cost function in the form

$$
J_{\text {polc }}(u)=\left(s+D \widehat{x}-G_{x} u\right)^{T}\left\langle n n^{T}\right\rangle\left(s+D \widehat{x}-G_{x} u\right)+(\widehat{x}+u)^{T}\left\langle x x^{T}\right\rangle^{-1}(\widehat{x}+u),
$$

where (on account of Eq. (18)) the matrix $D$ is defined by the equation

$$
D=G_{a} F_{x}-G_{x}
$$

The value of $r$ that minimizes $J_{\text {polc }}$ may then be evaluated as

$$
r=\left(G_{x}^{T}\left\langle n n^{T}\right\rangle^{-1} G_{x}+\left\langle x x^{T}\right\rangle^{-1}\right)^{-1}\left[G_{x}^{T}\left\langle n n^{T}\right\rangle^{-1} s+\left(G_{x}^{T}\left\langle n n^{T}\right\rangle^{-1} D-\left\langle x x^{T}\right\rangle^{-1}\right) \widehat{x}\right] .
$$

This formula is highly similar to the standard open-loop minimum variance reconstruction algorithm, with the addition of the second term within the square brackets that depends upon the previous value of the turbulence estimate $\widehat{x}$. This modification does not degrade the computational efficiency of the algorithm, since this term may be evaluated using sparse matrix operations.

The POLC algorithm has several attractive features, including its computational efficiency and very close relationship to the classical minimum variance reconstructor. On the other hand, it is not yet clear how sensitive this approach may be to uncertainties in the values of $a$ and $G_{a}$ due to hysteresis, calibration errors, and other implementation error sources. For this reason we have also investigated a second approach that is a genuinely closed loop control algorithm.

\subsection{Approximate closed-loop regularization (ACLR)}

The idea behind this approach is to adjust the atmospheric turbulence covariance matrix $\left\langle x x^{T}\right\rangle$ appearing in the definition of the classical minimum variance estimator so that it approximates the statistics of the residual reconstruction errors remaining in a closed-loop AO system. Ideally, we would like to compute and apply the optimum Kalman filter for this problem, but we are strongly concerned that this approach will be computationally impractical for very high order MCAO systems on future giant telescopes. Our approach is to use an approximate covariance matrix that yields a computationally efficient reconstruction algorithm; we hope that this approximation to the regularizing term is close enough to yield satisfactory performance in closed loop, but this assertion needs to be verified via Monte Carlo simulations.

Our first approximation to the atmospheric turbulence covariance matrix is based upon the assumption that the dominant source of residual wavefront error in the closed-loop AO system is time delay. The dynamic behavior of the $\mathrm{AO}$ control loop is governed by the equation

$$
a(z)=F_{x} g(z) E_{x}\left[G_{x} x(z)-G_{a} a(z)+n(z)\right] .
$$

Solving for the DM actuator command vector $a(z)$ obtains

$$
a(z)=\left[I+F_{x} g(z) E_{x} G_{a}\right]^{-1} F_{x} g(z) E_{x}\left[G_{x} x(z)+n(z)\right]
$$


for the closed-loop transfer function between the turbulence profile $x$ and the DM actuator command vector $a$. To eliminate all sources of wavefront error besides time delay, assume that $n(z) \equiv 0$, fitting error is negligible $\left(\operatorname{dim} a=\operatorname{dim} x\right.$ and $\left.G_{a}=G_{x}\right)$, and that the wavefront reconstruction algorithm has been perfectly calibrated $\left(F_{x} E_{x} G_{a} \equiv I\right)$. These simplifications yield the result

$$
x(z)-a(z)=[1+g(z)]^{-1} x(z)
$$

for the residual error in the AO control loop. Simplifying further, this can be approximated as a simple time delay of the form

$$
\begin{aligned}
x(t)-a(t) & \approx x(t)-x(t-\tau) \\
& \approx \tau x^{\prime}(t) \\
& \equiv x_{d}(t),
\end{aligned}
$$

where $\tau$ is the characteristic time delay for the AO control loop.

Using this time-delay-only model for the residual wavefront error in a closed-loop AO system, the merit function for defining the minimum variance (or ML) reconstruction algorithm becomes

$$
J_{a c l r}(u)=\left(s-G_{x} u\right)^{T}\left\langle n n^{T}\right\rangle^{-1}\left(s-G_{x} u\right)+u^{T}\left\langle x_{d} x_{d}^{T}\right\rangle^{-1} u .
$$

The estimate of the residual phase error $r$ at each control loop cycle is given by

$$
\begin{aligned}
r & =\arg \min _{u} J_{a c l r}(u) \\
& =\left(G_{x}^{T}\left\langle n n^{T}\right\rangle^{-1} G_{x}+\left\langle x_{d} x_{d}^{T}\right\rangle^{-1}\right)^{-1} G_{x}^{T}\left\langle n n^{T}\right\rangle^{-1} s .
\end{aligned}
$$

This takes the same form as the standard open-loop minimum variance estimator with the turbulence covariance matrix $\left\langle x x^{T}\right\rangle$ replaced by $\left\langle x_{d} x_{d}^{T}\right\rangle$, and the reconstruction algorithm will be computationally efficient to implement if we can find a good sparse approximation to the inverse of the latter matrix.

Since $x_{d}$ is proportional to the time derivative of $x$, it follows from the Taylor (or frozen flow) hypothesis that the spatial power spectrum of $x_{d}$ is proportional to $\kappa^{2} \kappa^{-11 / 3}=\kappa^{-5 / 3}$ if the direction of the wind is assumed to be random and uniformly distributed from 0 to $2 \pi$. In the same manner that we approximated $\kappa^{-11 / 3}$ as $\kappa^{-4}$ for the open-loop minimum variance estimator, ${ }^{16}$ we now propose to approximate the spatial power spectrum of $x_{d}$ with a $\kappa^{-2}$ power law. It may be shown that this is equivalent to approximating the inverse covariance matrix $\left\langle x_{d} x_{d}^{T}\right\rangle^{-1}$ as $\alpha D^{T} D$, where $\alpha$ is a scale factor and $D$ is a discrete (and sparse) form of the gradient (or Differentiation) operator. The resulting wavefront reconstruction algorithm has the same computational efficiency as the open-loop minimum variance MCAO reconstruction algorithm developed previously because the regularization terms $\alpha D^{T} D$ and $\alpha C^{T} C$ are equivalently sparse. Of course, Monte Carlo simulations are sorely needed to evaluate how the all of the approximations made in deriving the regularization term impact its stability and performance.

\subsection{A blended reconstruction algorithm}

The pair of merit functions $J_{\text {polc }}(u)$ and $J_{a c l r}(u)$ that determine the pair of reconstruction algorithms described above are so similar that it is irresistable to consider them as special cases of the "blended" merit function

$$
J_{b}(u ; \alpha)=\alpha J_{p o l c}(u)+(1-\alpha) J_{a c l r}(u) .
$$

This function is still quadratic in $u$, and one final least squares minimization argument yields the result

$$
\begin{aligned}
r= & \arg \min _{u} J_{b}(u ; \alpha) \\
= & {\left[G_{x}^{T}\left\langle n n^{T}\right\rangle^{-1} G_{x}+\alpha\left\langle x x^{T}\right\rangle^{-1}+(1-\alpha)\left\langle x_{d} x_{d}^{T}\right\rangle^{-1}\right]^{-1} } \\
& \quad \times\left[G_{x}^{T}\left\langle n n^{T}\right\rangle^{-1} s+\alpha\left(G_{x}^{T}\left\langle n n^{T}\right\rangle^{-1} D-\left\langle x x^{T}\right\rangle^{-1}\right) \widehat{x}\right] .
\end{aligned}
$$


Table 1. Atmospheric turbulence profile used for AO simulations. This table lists the altitudes, relative turbulence weights, and windspeeds for a six-layer atmospheric turbulence profile derived from thermosonde and generalized SCIDAR measurements at Cerro Pachon, Chile. The overall profile was scaled to yield $r_{0}=16 \mathrm{~cm}$ at $\lambda=0.5 \mu \mathrm{m}$. The corresponding isoplanatic angle is $\theta_{0}=12.85 \mu \mathrm{rad}$, and the Greenwood frequency is $f_{g}=27.5 \mathrm{~Hz}$.

\begin{tabular}{|r|r|r|r|}
\hline Layer & Altitude, $\mathrm{km}$ & Layer weight & Windspeed, $\mathrm{m} / \mathrm{s}$ \\
\hline 1 & 0.00 & 0.652 & 5.0 \\
2 & 2.58 & 0.172 & 13.0 \\
3 & 5.16 & 0.055 & 20.0 \\
4 & 7.73 & 0.025 & 30.0 \\
5 & 12.89 & 0.074 & 20.0 \\
6 & 15.46 & 0.022 & 10.0 \\
\hline
\end{tabular}

\section{SIMULATION TEST CASES}

This section summarizes the atmospheric and AO parameters we have used in initial closed-loop simulations of the above wavefront reconstruction algorithms for sample NGS AO and LGS MCAO system configurations on 8- and 32 meter class telescopes.

Table 1 summarizes the atmospheric turbulence profile used in these simulations. This profile is a six-layer fit to thermosonde and generalized SCIDAR measurements collected at Cerro Pachon, Chile, the site of the Gemini-South telescope. ${ }^{18}$ The profile has been scaled to obtain a $r_{0}$ of $16 \mathrm{~cm}$ at a wavelength of $0.5 \mu \mathrm{m}$, which corresponds to roughly median seeing. The value of the isoplanatic angle $\theta_{0}$ is $2.65 \operatorname{arc~sec}$, or $12.85 \mu \mathrm{rad}$. The wind velocities have been selected to match the general shape of the median wind profile at Cerro Pachon, and provide a representative Greenwood Frequency, $f_{g}$, of $27.5 \mathrm{~Hz}$.

The AO parameters for the 8- and 32 meter MCAO systems tested against this atmospheric profile are, for the most part, borrowed from the baseline design for a MCAO system at Gemini-South. ${ }^{4}$ The science field-ofview to be corrected is a one-arc-minute square. AO system performance is sampled the center, edges, and corners of this square field, and the field-averaged residual mean-square phase error is estimated by combining the errors at these nine evaluation points using Simpson's rule in two dimensions. Three deformable mirrors are located conjugate to ranges of $0.0,5.15$, and $10.30 \mathrm{~km}$, which are modest adjustment to the Gemini-South values of $0.0,4.5$, and $9.0 \mathrm{~km}$. The interactuator spacings on the three mirrors are equivalent to $0.5,0.5$, and $1.0 \mathrm{~m}$ at the telescope primary mirror.

The three DM's are controlled using measurements from five higher-order wavefront sensors viewing sodium laser guidestars at a range of $90 \mathrm{~km}$. For the 8 -meter MCAO system, the 5 guidestar are located at the center and four corners of the 1 arc-minute-square science field of view. For the 32-meter MCAO system, the 4 outer guidestars have been radially displaced outwards to a distance of $\sqrt{2} \cdot 46=65$ arc sec from the center of the field to measure a larger fraction of the higher turbulence layers. The subaperture width for each WFS is equivalent to $0.5 \mathrm{~m}$ at the telescope primary mirror. The full aperture tilt modes of the LGS WFS measurements are unreliable since the exact positions of the guidestars on the sky are variable and unknown, so four natural guide stars located at the midpoints of the edges of the field are all included for full aperture tip/tilt sensing. (This deviation from the 3 NGS proposed for Gemini-South yields symmetric performance over the square field of view, which simplifies the performance analysis.)

We have also simulated a conventional 8-meter natural guide star (NGS) AO system with a single DM, a single on-axis guidestar, and the same actuator/subaperture densities. For this case, the atmospheric model was simplified to a single phase screen in the aperture plane with a wind velocity of $10 \mathrm{~m} / \mathrm{sec}$.

There are a total of 2240 (33320) LGS WFS measurements for the MCAO simulations with $D=8 \mathrm{~m}$ $(D=32 \mathrm{~m})$, and the total number of DM actuators is 789 (8449). For the NGS AO simulation, there is a total of 448 WFS measurements and 257 DM actuators. 
The simulations assume a higher-order WFS measurement error of 0.02 arc seconds RMS, which corresponds to a bright guide star and is perhaps 2 or 3 times smaller than the best we might hope to achieve for sodium LGS MCAO with reasonable laser powers and beam qualities. The noise levels for the NGS tip/tilt sensors have been scaled down by the ratio of the subaperture size to the full aperture diameter (i.e., a factor of $8 / 0.5=16$ for $D=8 \mathrm{~m}$ or a factor of 64 for $D=32 \mathrm{~m}$ ) to yield the same phase difference measurement error across the full aperture.

Finally, the simulation cycle time was $1 / 800$ seconds, which matches the baseline design for the GeminiSouth MCAO system. The servo filter $\mathrm{g}(\mathrm{z})$ determining the temporal dynamics of the AO control loop was defined by the finite difference equation

$$
\widehat{x}(n)=\frac{1}{2} \widehat{x}(n-1)+\frac{1}{2} \widehat{x}(n-2)+\frac{1}{2} r(n-2),
$$

which is equivalent to the transfer function

$$
\widehat{x}(z)=\frac{1}{2} z^{2}\left(1-\frac{1}{2} z-\frac{1}{2} z^{2}\right)^{-1} r(z)
$$

in the $z$-transform domain. Note the use of the term $r(n-2)$, not $r(n-1)$, appearing in Eq. (33) above; this extra cycle of temporal latency in the control loop is an approximation to the additional time delay induced by reading out the WFS detectors. The corresponding closed-loop impulse response function for a single-input, single-output servo system (where $x$ is the input disturbance and $r=x-\widehat{x}$ is the residual error and the sensor measurement) would be

$$
\begin{aligned}
\widehat{x}(z) / x(z) & =\frac{1}{2} z^{2} /\left(1-\frac{1}{2} z\right) \\
& =\frac{1}{2} z^{2} \sum_{n=0}^{\infty}(z / 2)^{n} .
\end{aligned}
$$

It can be shown that the $-3 \mathrm{~dB}(0 \mathrm{~dB})$ closed loop bandwidth for this control law is about $30(50) \mathrm{Hz}$, modestly higher than the Greenwood frequency of $27.5 \mathrm{~Hz}$ used in the simulation. ${ }^{4}$

\section{SAMPLE SIMULATION RESULTS}

This section summarizes a sample of the initial closed-loop simulation results we have obtained for the 8- and 32-meter class NGS AO and LGS MCAO systems described in section 5 above. In these early adventures we have been more interested in evaluating the stability properties, relative performance, and execution times for the reconstruction algorithms, as opposed to fully characterizing and optimizing the performance of AO systems used as test cases.

Fig. 1 plots the simulation results obtained for the simplest case considered, a NGS AO system with 257 actuators and 448 WFS measurements on an 8-meter telescope. The figure plots the log of the mean-square phase variance vs. time for (i) the input wavefront disturbance, (ii) the residual wavefront error for the standard minimum variance reconstructor applied naively in closed loop, (iii) the residual wavefront error for the ACLR (approximate closed-loop regularization) reconstruction algorithm, and (iv) the residual wavefront error for the POLC (Pseudo open-loop control) reconstructor. Reconstructors (ii) and (iii) are unstable and diverge after no more than 50 to 100 iterations, even though they initially provide a near-optimal solution after the first few cycles. It may be interesting to note that the ACLR algorithm diverges significantly less rapidly than the standard minimum variance reconstructor, but we have not yet investigated whether alternate forms of this approach might yield stable performance. In contrast, the performance achieved with the POLC approach is stable and near optimal. This is as should be expected in a problem with (i) a relatively low wind speed and (ii) ideal knowledge of the DM command vector and the DM-to-WFS influence matrix.

Fig. 2 illustrates the closed-loop MCAO simulation results obtained with the POLC reconstruction algorithm. Just as with the NGS AO case, the algorithm is stable and yields performance that is very similar to the openloop estimation accuracy of the standard minimum variance reconstructor.

The time required per reconstruction is about 10 and 330 seconds for the 8 and 32 meter MCAO simulations, respectively. This is for 10 complete iterations of preconditioned conjugate gradients, ${ }^{5-7}$ which could probably be reduced somewhat with negligible impact upon performance. 


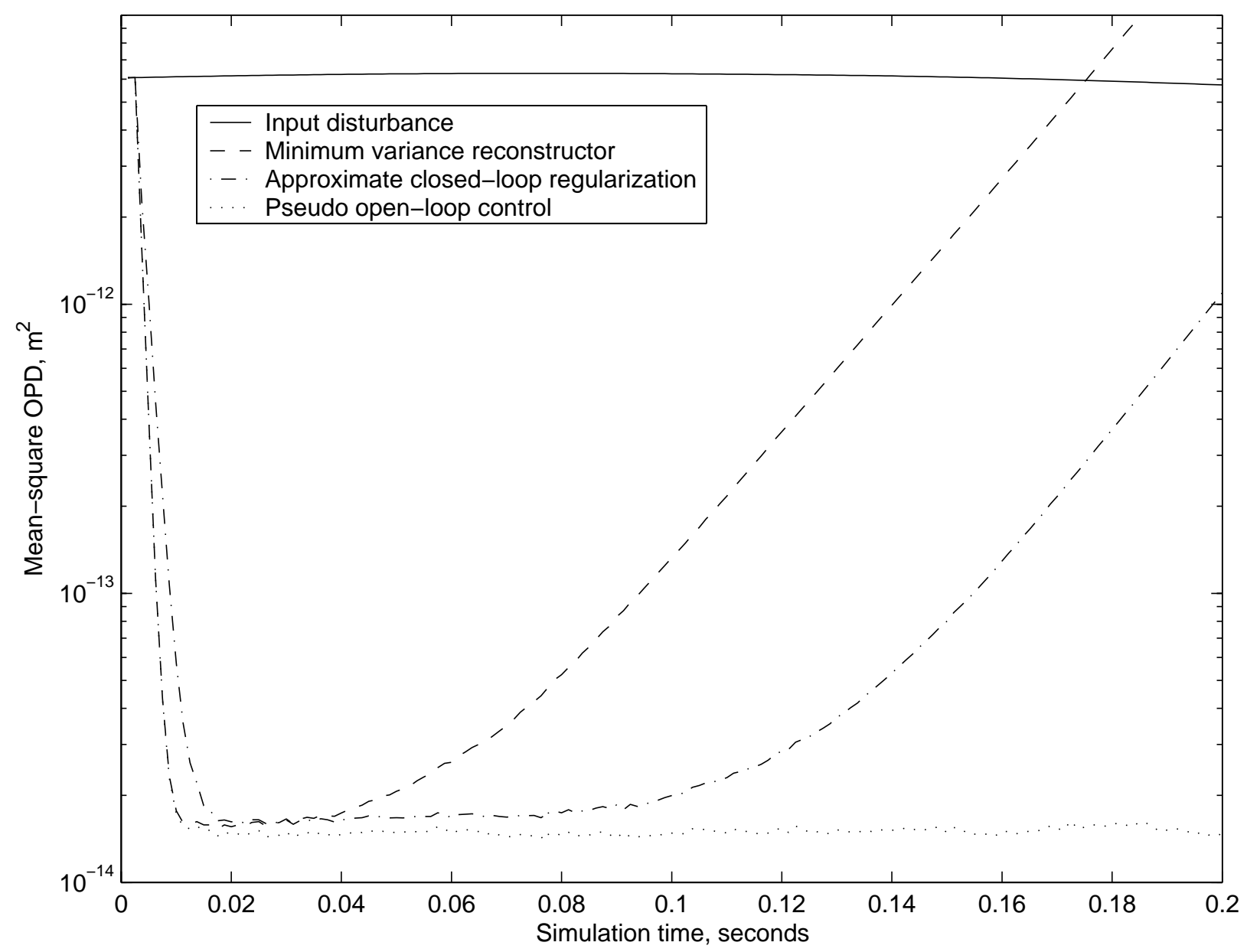

Figure 1. Closed-loop simulation results for an 8-meter NGS AO system

This figure plots the mean-square phase estimation error vs. time for an order $16^{2}$ NGS AO system and three different wavefront reconstruction algorithms as described in sections 4 and 5 above.

\section{SUMMARY AND PLANS}

The very high-order MCAO systems now being proposed for future giant telescope will require new wavefront reconstruction concepts due to the large number of DM actuators and WFS subapertures. Current methods for computing stable wavefront reconstructors for closed-loop AO system have computational complexity $O\left(n^{3}\right)$, and consequently do not appear to be applicable. In this paper, we have investigated two approaches to adapting the standard minimum variance reconstructor, which is optimized for wavefront estimation in open loop, into a computationally efficient form that will remain stable and provide acceptable performance in closed loop. The first approach, pseudo-open loop control (POLC), assumes that accurate knowledge of the DM actuator commands and the DM-to-WFS influence matrix is available to transform closed-loop WFS measurements into the corresponding open-loop values so that the standard minimum variance reconstructor may be applied. The second method, approximate closed-loop regularization (ACLR), adapts the regularization term appearing in the definition of the minimum variance reconstructor to (approximately) represent the residual errors in a closed loop AO system.

The POLC reconstruction algorithm obtained stable results and good closed-loop performance in initial simulations of NGS AO and LGS MCAO system on 8- and 32-meter telescopes. The ACLR algorithm is unstable, 

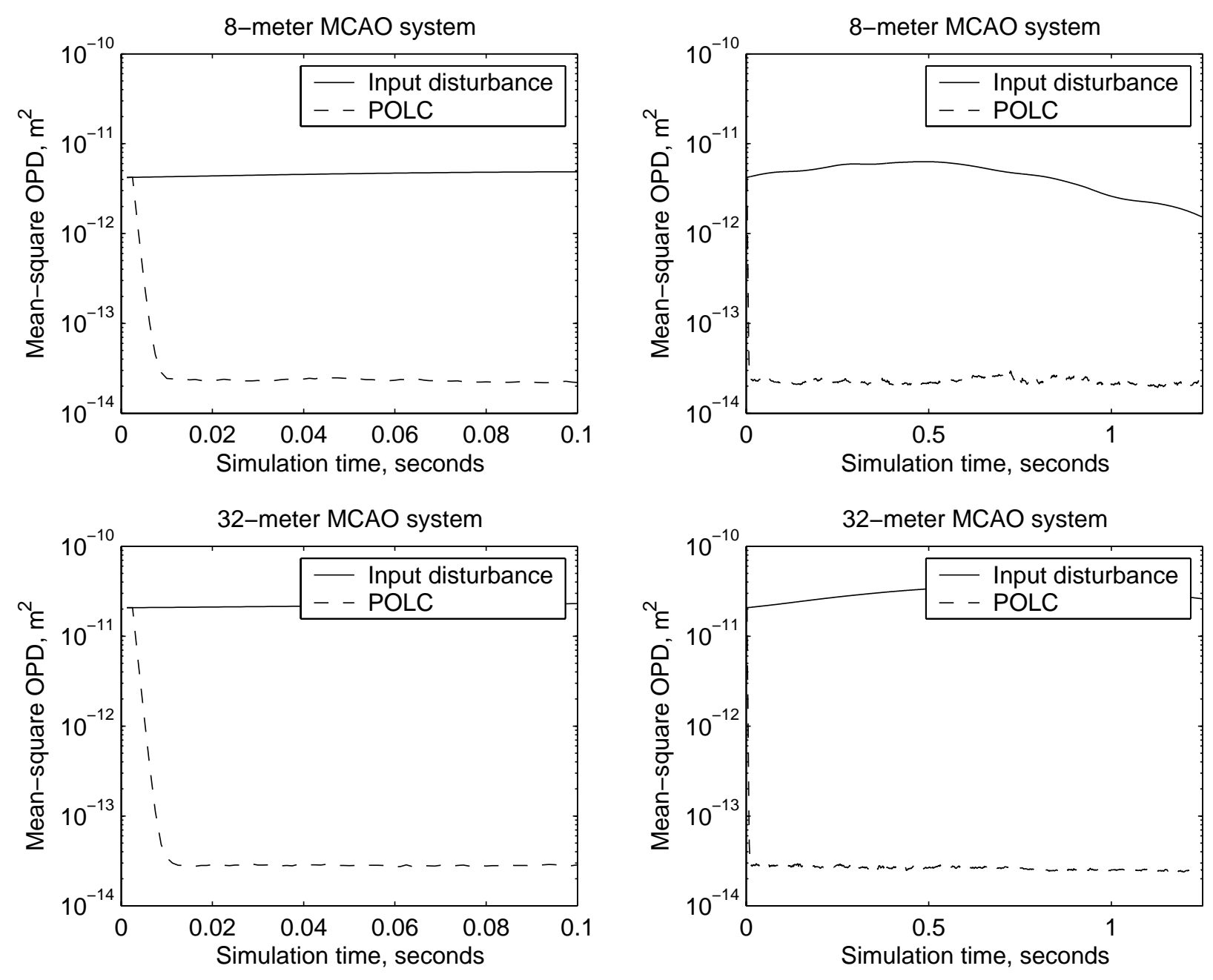

Figure 2. Closed loop simulation results for 8- and 32-meter MCAO systems

This figure plots the mean-square phase estimation error vs. time for the pseudo open loop control (POLC) reconstruction algorithm and the 8- and 32-meter MCAO system configurationsdescribed in section 5 above. The results are plotted on two time scales for each system to better illustrate the convergence behavior and long-term stability of the control loop.

although it diverges more slowly than the standard minimum variance reconstruction algorithm applied naively in closed loop.

We now plan to assess the sensitivity of the POLC reconstruction algorithm to a variety of calibration errors, including: non-common path wavefront aberrations with non-Kolmogorov statistics, variations in WFS tilt measurement gain due to changes in atmospheric seeing and the dimensions of the laser guide stars, and DM-to-WFS misregistration errors. We will then install the new reconstructor into a wave optics propagation simulation for futher study of MCAO system performance issues on ELT's, including the impact of extended laser guide stars.

\section{ACKNOWLEDGMENTS}

The New Initiatives Office is a partnership between two divisions of the Association of Universities for Research in Astronomy (AURA), Inc.: The National Optical Astronomy Observatory (NOAO) and the Gemini Observatory. NOAO is operated by AURA under a cooperative agreement with the National Science Foundation 
(NSF). The Gemini Observatory is operated by AURA under a cooperative agreement with the NSF on behalf of the Gemini partnership: the National Science Foundation (United States), the Particla Physics and Astronomy Research Council (United Kingdom), the National Research Council (Canada), CONICYT (Chile), the Australian Research Council (Australia), CNPq (Brazil) and CONICET (Argentina).

\section{REFERENCES}

1. J. E. Nelson, "CELT Rocks," in Future Giant Telescopes, James Roger P. Angel and Roberto Gilmozzi, ed., SPIE Proceedings Vol. 4840 47-59 (2002).

2. S. E. Strom, L. M. Stepp, M. Mountain, and B. Gregory, "Giant segmented mirror telescope: a point design based on science drivers," in Future Giant Telescopes, James Roger P. Angel and Roberto Gilmozzi, ed., SPIE Proceedings Vol. 4840 116-128 (2002).

3. P. Dierickx, J. Beckers, E. Brunetto, R. Gilmozzi, F. Koch, E. Marchetti, G. J. Monnet, M. Quattri, M. S. Sarazin, J. Spyromilio, and N. Yaitskova, "Eye of the beholder: designing the OWL," in Future Giant Telescopes, James Roger P. Angel and Roberto Gilmozzi, ed., SPIE Proceedings Vol. 4840 151-170 (2002).

4. Gemini-South MCAO PDR documentation, http://www.gemini.edu/sciops/instruments/adaptiveOptics/AOarchive.html

5. B. L. Ellerbroek, L. Gilles, and C. R. Vogel, "Computationally efficient wavefront reconstruction algorithms for simulations of multiconjugate adaptive optics on giant telescopes," in Adaptive Optical Systems Technologies II, Peter L. Wizinowich, ed., SPIE Proceedings Vol. 4839 989-1000 (2002).

6. L. Gilles, B. L. Ellerbroek, and C. R. Vogel, "Layer-oriented multigrid wavefront reconstruction algorithms for multiconjugate adaptive optics," in Adaptive Optical Systems Technologies II, Peter L. Wizinowich, ed., SPIE Proceedings Vol. 4839 1011-1022 (2002).

7. L. Gilles, B. L. Ellerbroek, and C. R. Vogel, "Order N sparse minimum-variance open-loop reconstructor for extreme adaptive optics," Optics Letters (in press).

8. B. L. Ellerbroek, L. Gilles, and C. R. Vogel, "Numerical Simulations of Multi-Conjugate Adaptive Optics Wavefront Reconstruction on Giant Telescopes," Appl. Opt. (in press).

9. B. L. Ellerbroek, "First order performance evaluation of adaptive-optics systems for atmospheric turbulence compensation in extended field-of-view astronomical telescopes," J. Opt. Soc. Am. A 11, 783-805 (1994).

10. B. Le Roux, J. M. Conan,C. Kulcsar, H-F. Raynaud, L. M. Mugnier, T. Fusco, "Optimal control law for multi-conjugate adaptive optics," in Adaptive Optical Systems Technologies II, Peter L. Wizinowich, ed., SPIE Proceedings Vol. 4839 878-889 (2002).

11. D. T. Gavel and W. Wiberg, "Towards strehl-optimizing adaptive optics controllers," in Adaptive Optical Systems Technologies II, Peter L. Wizinowich, ed., SPIE Proceedings Vol. 4839 890-901 (2002).

12. R. Flicker, F. J. Rigaut, and B. L. Ellerbroek, "Comparison of multiconjugate adaptive optics configurations and control algorithms for the Gemini South 8-m telescope," in Adaptive Optical Systems Technology, Peter L. Wizinowich, ed., SPIE Proceedings Vol. 4007 1022-1031 (2000).

13. T. Fusco, J.-M. Conan, G. Rousset, L. M. Mugnier, and V. Michau, "Optimal wave-front reconstruction strategies for multi-conjugate adaptive optics," J. Opt. Soc. Am. A 18, 2527-2583 (2001).

14. E. P. Wallner, "Optimal wave-front correction using slope measurements," J. Opt. Soc. Am. 73, 1771-1776 (1983).

15. D. C. Johnston and B. M. Welsh, "Analysis of multi-conjugate adaptive optics," J. Opt. Soc. Am. A 11, 394-408 (1994).

16. B. L. Ellerbroek, "Efficient computation of minimum-variance wave-front reconstructors with sparse matrix techniques," J. Opt. Soc. Am. A 19, 1803-1816 (2002).

17. L. Gilles, C. R. Vogel, and B. L. Ellerbroek, "A multigrid preconditioned conjugate gradient method for large scale wavefront reconstruction," J. Opt. Soc. Am. A 19, 1817-1822 (2002).

18. J. Vernin, A. Agabi, R. Avila, M. Azouit, R. Conan, F. Martin, E. Masciadri, L. Sanchez, and A. Ziad, "1998 Gemini site testing campaign: Cerro Pachon and Cerro Tololo," Gemini Doc. RTP-AO-G0094 (Gemini Observatory, Hilo, Hawaii, 2000). 